\title{
Role of Gut-Related Peptides and Other Hormones in the Amelioration of Type 2 Diabetes after Roux-en-Y Gastric Bypass Surgery
}

\author{
Mirella P. Hage, ${ }^{1}$ Bassem Safadi, ${ }^{2}$ Ibrahim Salti, ${ }^{1}$ and Mona Nasrallah ${ }^{1}$ \\ ${ }^{1}$ Division of Endocrinology, Department of Internal Medicine, American University of Beirut-Medical Center, \\ P.O. Box 11-0236/D23 Riad El-Solh, Beirut 1107 2020, Lebanon \\ ${ }^{2}$ Department of Surgery, American University of Beirut-Medical Center, P.O. Box 11-0236/D23, Riad El-Solh, \\ Beirut 1107 2020, Lebanon
}

Correspondence should be addressed to Mona Nasrallah, mn36@aub.edu.lb

Received 10 January 2012; Accepted 6 February 2012

Academic Editors: J. E. Gunton, D. Iafusco, and N. Tentolouris

Copyright (c) 2012 Mirella P. Hage et al. This is an open access article distributed under the Creative Commons Attribution License, which permits unrestricted use, distribution, and reproduction in any medium, provided the original work is properly cited.

\begin{abstract}
Bariatric surgery is currently the most effective and durable therapy for obesity. Roux-en-Y gastric bypass surgery, the most commonly performed procedure worldwide, causes substantial weight loss and improvement in several comorbidities associated with obesity, especially type 2 diabetes. Several mechanisms are proposed to explain the improvement in glucose metabolism after RYGB surgery: the caloric restriction and weight loss per se, the improvement in insulin resistance and beta cell function, and finally the alterations in the various gastrointestinal hormones and adipokines that have been shown to play an important role in glucose homeostasis. However, the timing, exact changes of these hormones, and the relative importance of these changes in the metabolic improvement postbariatric surgery remain to be further clarified. This paper reviews the various changes post-RYGB in adipokines and gut peptides in subjects with T2D.
\end{abstract}

\section{Introduction}

The epidemic of obesity continues to increase, followed in close parallel by T2D, and the World Health Organization estimates show that by 2015 , around 2.3 billion adults will be overweight and greater than 700 million will be obese [1]. Recommendations to achieve weight loss include primarily lifestyle measures such as dietary therapy and exercise, limited pharmacological treatment, and bariatric surgery. Bariatric surgery has proven so far to be the most effective and durable treatment option for both the excess weight and the related comorbidities $[2,3]$. Strong evidence has revealed that in addition to inducing major weight loss, bariatric surgery further ameliorates diabetes, hypertension, and dyslipidemia [4]. Of those with T2D, 78\% had complete resolution following surgery and diabetes improved or resolved in $86.6 \%$ of patients. The greatest effect on weight loss and diabetes resolution was seen in patients undergoing biliopancreatic diversion/duodenal switch followed by gastric bypass and then banding procedures [5].
Among the various techniques in bariatric surgery, RYGB is the most common bariatric surgery performed worldwide and is considered by many surgeons as the "gold standard" procedure [6]. The RYGB operation was developed in the 1960's following observations of weight loss after gastric resection for peptic ulcer disease. Surgeons worked on multiple alterations of the operation and deduced that for effective weight reduction, the stomach size needs to be reduced to less than $50 \mathrm{mLs}$. This small part of the stomach that remains in continuity with the digestive tract is referred to as the gastric pouch, whereas the majority of the stomach and the duodenum are excluded and are no longer in direct contact with food. The gastric pouch is then reattached to the small intestines using either staples or sutures, and this connection is referred to as the stoma. The preferred way to connect the pouch to the small intestine is via a Roux-yconfiguration as shown in Figure 1. In the RYGB, the food goes across the pouch into the "alimentary limb", whereas the biliary and pancreatic juices flow a distance away from 


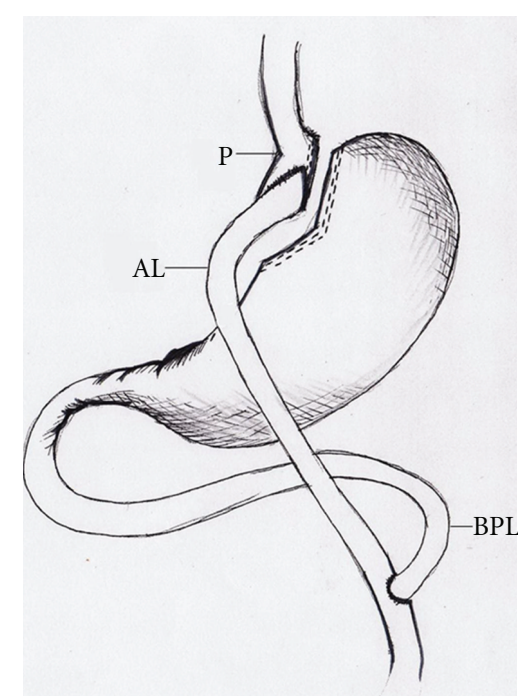

FIGURE 1: Roux-en-Y gastric bypass. P: gastric pouch. AL: alimentary limb. BPL: biliopancreatic limb.

the pouch to form what is referred to as the "biliopancreatic limb" to minimize the harmful effects of "bile reflux" [7].

Several studies have demonstrated the dramatic effect of RYGB on T2D occurring as early as 6 days postoperatively long before major weight loss has occurred [8]. Elucidating the mechanisms of improvement of diabetes after RYGB may lead to a better understanding of the pathophysiology of T2D and guide the search for novel therapies. Hypothesis linking the early and rapid metabolic improvement to bariatric surgery have focused on hormonal changes, namely, adipokines and gut peptides. Therefore, the purpose of this paper is to critically review the recent data and clinical studies addressing the changes in gut-related peptides and other hormones after RYGB surgery and the resulting alterations in metabolic profile.

\section{Literature Search}

A Pubmed search through the English Literature was conducted from 1979 to 2010 using various combinations of the following key words: "adiponectin", "amylin", "bariatric surgery", "gastric bypass", "gastrointestinal hormones", "GLP-1", "ghrelin", "gut hormones”, “insulin”, "leptin”, "metabolic surgery”, "obesity”, “oxyntomodulin”, "peptide YY" (PYY), and "Roux-en-Y gastric bypass" (RYGB). Only longitudinal and cross-sectional studies assessing hormonal changes after RYGB surgery in obesity and diabetes from year 2000 to 2010 were identified and included due to paucity of studies addressing this issue before year 2000 .

\section{Mechanisms of Improvement of Diabetes after RYGB Surgery}

Weight loss per se and the decrease in fat mass induced by bariatric surgery reduce insulin resistance through the direct and indirect effects of adipocytokines and through the fall in lipid content in both liver and muscle. Furthermore, caloric restriction imposed by bariatric surgery allows the beta-cells to rest and they are thus minimally challenged. A significant improvement in glucose homeostasis that is independent of weight loss can be achieved by following a very lowcaloric diet $[9,10]$. One study by Henry et al. showed that a $330 \mathrm{cal} /$ day diet resulted in reduction in fasting plasma glucose from $297 \mathrm{mg} / \mathrm{dL}$ to $138 \mathrm{mg} / \mathrm{dL}$ over 40 days with $87 \%$ of this drop occurring in the first 10 days [10]. However, the effects of weight reduction and caloric restriction alone do not account for the dramatic and sustained effects of bariatric surgery on diabetes, long after negative caloric balance had ceased. Two hypotheses have been proposed to explain how bariatric surgery improves diabetes early on.

(1) Hind gut hypothesis: this hypothesis holds that diabetes control results from the rapid delivery of nutrients to the lower intestine overstimulating the $\mathrm{L}$ cells to release gut hormones such as GLP-1, peptide YY, and oxyntomodulin. These hormones exert anorectic and insulinotropic effects to various extents thus improving glucose homeostasis [11, 12].

(2) Foregut hypothesis: in normal conditions, the passage of nutrients along the proximal bowel stimulates the production of an unidentified factor with antiincretin properties responsible for insulin resistance and abnormal glycemic control. Thus, the exclusion of the proximal intestine would reduce the production of anti-incretins and would therefore increase insulin secretion and action and improve diabetes [12-14].

Rubino et al. supported the foregut hypothesis as an explanation for the improvement in glucose homeostasis after RYGB surgery. They showed that excluding the proximal intestine in Goto-Kakizaki (GK) diabetic rats that have undergone gastrojejunostomy ameliorated their diabetes compared to rats with an intact duodenal passage. Conversely, in rats that had undergone duodenal-jejunal bypass, restoration of their duodenal passage reestablished their impaired glucose tolerance [15]. A recent study by Knop suggested a possible role for glucagon or gut-derived glucagonotropic signaling as diabetogenic signal of the foregut hypothesis [14].

Since RYGB significantly changes the anatomy of the gastrointestinal tract, alteration in the secretion of several gut peptides ensues. These hormones are involved in appetite regulation and energy balance and have been implicated in glucose homeostasis as well. In the next section follows a detailed review of individual peptides and hormones.

\section{Gut Hormones}

4.1. Ghrelin. Ghrelin is a 28 aminoacid peptide secreted predominantly by the X/A-like enteroendocrine cells of the fundus of the stomach [16]. Plasma ghrelin levels rise nearly twofold before a meal and fall within one hour after eating [17]. It is the only known circulating orexigen. Ghrelin circulates in two different forms: acylated ghrelin and desacylated ghrelin [18]. Acyl-ghrelin accounts for less 
than $10 \%$ of the total circulating ghrelin. It binds to GHSR1a receptor and stimulates food intake as well as GH secretion [19]. Des-acyl ghrelin, the major circulating form of ghrelin, does not bind to GHSR1a receptor but it is not biologically inactive. However, it has been shown to counteract the effects of acyl-ghrelin on insulin secretion and glucose metabolism in humans [20] and reduce food intake in mice [21].

How does ghrelin contribute to diabetes resolution after RYGB surgery?

Ghrelin has been shown to increase levels of GH [22], cortisol, and epinephrine [23], three counter regulatory hormones that oppose insulin action. It decreases as well secretion of the insulin sensitizing hormone adiponectin [24]. In addition, ghrelin suppresses intracellular insulin signaling in cultured hepatocytes [25] impairing hepatic insulin sensitivity. Furthermore, ghrelin could influence insulin secretion by a direct effect on the pancreas as the ghrelin receptor GHSR1-a is expressed in various tissues including the pancreatic islets $[26,27]$. Exogenous ghrelin administration decreases insulin secretion in both human and animal studies $[28,29]$. Some studies report improved glucose disposal by muscle under ghrelin; however, the effect of ghrelin on the liver is that of insulin insensitivity so that the overall riding effect is that of an increase in plasma glucose [30, 31]. Therefore suppression of ghrelin after RYGB surgery is associated with improved glucose homeostasis.

Studies reporting changes in ghrelin levels after RYGB surgery have shown conflicting results (see Table 1). Ghrelin levels have been reported to decrease as early as during the intraoperative period following division of the stomach [32] or later [33-38]. Other studies have reported no change in ghrelin levels after RYGB surgery [34, 39-41].

4.2. Glucagonlike Peptide-1 (GLP-1). Glucagonlike peptide1 is a 30 aminoacid peptide secreted by the $\mathrm{L}$ cells of the distal ileum and colon in response to ingested nutrients. It enhances glucose-dependent insulin release and improves beta cell function $[42,43]$. Furthermore, it inhibits glucagon secretion, delays gastric emptying and indirectly decreases food intake [44]. Circulating GLP-1 has a short half-life of less than 2 minutes principally due to its inactivation by the plasma enzyme dipeptidyl peptidase-IV (DPP-IV) [45]. Chronic subcutaneous GLP-1 administration improved glycemic control and decreased body weight in type 2 diabetic patients [46]. In fact, GLP-1 R agonists, resistant to DPPIV inactivation, have been successfully used in the treatment of diabetes. Similarly, direct DPPIV inhibitors also improve glycemic control in T2D, although to a lesser extent, likely because endogenous GLP-1 levels are low in diabetes. Therefore, GLP-1 retains its insulinotropic property in diabetic subjects, but its circulating levels are decreased $[47,48]$.

In theory, GLP-1 should increase after RYGB due to the rapid nutrient delivery to the ileum where most of the $\mathrm{L}$ cells are located. Most data obtained regarding changes in GLP-1 after RYGB surgery have shown an increase [49-58], supportive of this theory, except for one study which showed a decrease in both controls and subjects [59]. Few others have shown no change $[41,60,61]$. A summary of the studies is presented in Table 2.

4.3. Glucose-Dependent Insulinotropic Polypeptide (GIP). Glucose-dependent insulinotropic peptide formerly known as gastric inhibitory polypeptide is a 42 aminoacid peptide that is secreted by the $\mathrm{K}$ cells of the duodenum and jejunum in response to ingested nutrients. It enhances glucosedependent insulin secretion [62] and promotes beta cell proliferation [63]. Higher levels of basal GIP as well as an increased early phase postprandial GIP response were seen in obese subjects compared to lean individuals [48]. In subjects with T2D, the overall effect of GIP seems to be in favor of hyperglycemia. In a recent study by Chia et al., exogenous administration of GIP raised glucose levels in type 2 diabetic patients in both early and late postprandial phases [64]. One mechanism is the impaired insulinotropic action of GIP which has been observed particularly during the late phase of insulin secretion [47]. This could be explained by a defective expression of GIP receptors as observed in Zucker diabetic fatty rats [65]. Secondly, although GIP is an insulinotropic hormone, an elevation of glucagon secretion with GIP infusion was observed in the early postprandial phase counteracting insulin glucose lowering effect. Thirdly, exogenous administration of GIP, as reported by Chia et al., worsened hyperglycemia in the late postprandial phase evoking a potential suppressive effect of GIP on GLP1 [64]. Furthermore, GIP may be directly implicated in fat metabolism and development of obesity by influencing insulin sensitivity of adipocytes. GIP promotes deposition of fat in adipose tissues and inhibits lipolysis [66, 67]. Mice lacking GIP receptors [68] or K cells [69]were protected from obesity when fed a high-fat diet, and young prediabetic $o b / o b$ mice treated with $\left(\mathrm{Pro}^{3}\right)$ GIP a specific and stable GIP receptor antagonist prevented the development of diabetes and related metabolic abnormalities in these rodents [70] Therefore, GIP receptor antagonists, by opposing GIP's anabolic action on adipose tissue, could represent a new treatment modality for obesity [71].

Since GIP is secreted by the proximal gut, bypassing the duodenum and part of the jejunum in the RYGB surgery is expected to result in a decrease in GIP secretion and therefore a more favorable glycemic milieu. Studies involving GIP and RYGB surgery have shown inconsistent results. Reduced levels postoperatively have been reported in some studies $[60,61]$ while others reported no change or an increase in GIP levels after surgery as shown in Table $3[49,50,72,73]$.

4.4. Oxyntomodulin (OXM). Oxyntomodulin is a 37 aminoacid peptide derived from proglucagon cosecreted with GLP-1 and PYY from the L cells of the distal gut in response to ingested nutrients [74]. Central and peripheral administration of OXM has been observed to reduce food intake in rats [75]. Infusion of OXM in humans prolonged gastric emptying, reduced pancreatic exocrine secretion, and decreased food intake $[76,77]$. Furthermore, subcutaneous administration of OXM decreased body weight in overweight and obese individuals [78]. However, the effect of exogenous 
TABLE 1: Ghrelin and RYGB surgery.

\begin{tabular}{|c|c|c|c|c|c|c|}
\hline Author/year & Type of study & Subjects & Preop BMI & $\%$ weight loss & $\mathrm{F} / \mathrm{U}$ time & Change in hormone \\
\hline $\begin{array}{l}\text { Geloneze et al. } \\
2003[33]\end{array}$ & $\begin{array}{l}\text { Prospective } \\
\text { controlled }\end{array}$ & $\begin{array}{l}28 \text { RYGB } \\
\text { surgery } \\
14 \text { T2D } \\
14 \text { NGT } \\
\text { lean group }\end{array}$ & $\begin{array}{c}56.3 \pm 10.2 \\
24.2 \pm 1.5\end{array}$ & $\begin{array}{c}\% \text { EWL } \\
67.4 \pm 13.4\end{array}$ & $1 \mathrm{y}$ & $\begin{array}{l}\text { Lower ghrelin levels in obese } \\
\text { compared to lean presurgery; } \\
\text { No difference in fasting ghrelin in } \\
\text { T2D and NGT before surgery; } \\
\text { Decrease in fasting ghrelin in both } \\
\text { T2D and NGT after surgery. }\end{array}$ \\
\hline Lin et al. 2004 [32] & $\begin{array}{l}\text { Prospective } \\
\text { controlled }\end{array}$ & $\begin{array}{l}34 \text { RYGB } \\
4 \text { VBG } \\
4 \text { ARS } \\
4 \text { lean ARS }\end{array}$ & $\begin{array}{l}47.0 \pm 0.7 \\
43.7 \pm 2.5 \\
40.0 \pm 2.0 \\
23.8 \pm 0.9\end{array}$ & NA & $\begin{array}{l}30 \mathrm{~min} \\
\text { postop }\end{array}$ & $\begin{array}{l}\text { Ghrelin higher in lean ARS } \\
\text { compared to pre-RYGB; } \\
\text { Decrease in ghrelin levels } \\
\text { post-RYGB to levels lower that } \\
\text { lean ARS; }\end{array}$ \\
\hline $\begin{array}{l}\text { Frühbeck et al. } \\
2004 \text { [37] }\end{array}$ & $\begin{array}{l}\text { Prospective } \\
\text { controlled }\end{array}$ & $\begin{array}{c}8 \text { RYGB } \\
8 \text { AGB } \\
8 \text { Conv } \\
6 \text { Total } \\
\text { gastrectomy }\end{array}$ & $\begin{array}{r}44.2 \pm 2.6 \\
44.8 \pm 1.6 \\
43.7 \pm 1.5 \\
29.9+1.1\end{array}$ & NA & $6 \mathrm{mo}$ & $\begin{array}{l}\text { At } 6 \text { mo, lower fasting ghrelin in } \\
\text { RYGB and gastrectomy groups } \\
\text { compared to AGB and conv group; } \\
\text { No differences in fasting ghrelin at } \\
6 \text { mos between RYGB group and } \\
\text { gastrectomy group. }\end{array}$ \\
\hline $\begin{array}{l}\text { Couce et al., } 2006 \\
{[34]}\end{array}$ & $\begin{array}{l}\text { Prospective } \\
\text { controlled }\end{array}$ & $\begin{array}{c}49 \text { obese }(30 \mathrm{~F}) \\
\text { RYGB } \\
19 \text { obese } \\
\text { (9 F) other } \\
\text { GI surgeries }\end{array}$ & $\begin{array}{c}50 \pm 5.3 \\
29.8 \pm 3.1\end{array}$ & NA & $\begin{array}{l}2 \mathrm{hr} \\
10 \mathrm{~d} \\
6 \mathrm{mo}\end{array}$ & $\begin{array}{l}\text { Decrease in fasting ghrelin at } 2 \mathrm{hr} \\
\text { in both groups compared to } \\
\text { preop; } \\
\text { Decrease in fasting ghrelin } 10 \mathrm{~d} \\
\text { postop in only RYGB group } \\
\text { compared to preop; } \\
\text { At } 6 \text { mo, no change in ghrelin } \\
\text { levels in both groups compared to } \\
\text { preoperative levels. }\end{array}$ \\
\hline $\begin{array}{l}\text { Morínigo et al. } \\
2008[35]\end{array}$ & $\begin{array}{l}\text { Prospective } \\
\text { controlled }\end{array}$ & $\begin{array}{l}25 \text { non diabetics } \\
\text { RYGB }(6 \mathrm{~F}) \\
6 \text { nonobese }(2 \\
\text { F) } \\
10 \text { severely } \\
\text { obese } \\
\text { T2D }(5 \mathrm{~F}) \text { RYGB }\end{array}$ & $\begin{array}{l}48.8 \pm 1.2 \\
49.2 \pm 2.0\end{array}$ & $43.0 \pm 2.3$ & 6 and $52 \mathrm{wk}$ & $\begin{array}{l}\text { Decrease in fasting plasma ghrelin } \\
\text { at } 6 \text { wk postop; } \\
\text { At } 52 \text { wk, plasma ghrelin returned } \\
\text { to baseline levels. }\end{array}$ \\
\hline $\begin{array}{l}\text { Karamanakos et al. } \\
2008[40]\end{array}$ & $\begin{array}{l}\text { Prospective } \\
\text { controlled }\end{array}$ & $\begin{array}{l}16 \text { RYGB }(12 \mathrm{~F}, \\
2 \mathrm{~T} 2 \mathrm{D}) \\
16 \text { LSG }(15 \mathrm{~F}, 1 \\
\text { IGT) }\end{array}$ & $\begin{array}{l}46.6 \pm 3.7 \\
45.1 \pm 3.6\end{array}$ & $\begin{array}{l}\% \text { EWL }^{\mathrm{a}} \\
60.5 \pm 10.7 \\
69.7 \pm 14.6\end{array}$ & $\begin{array}{l}1,3,6 \text { and } \\
12 \mathrm{mo}\end{array}$ & $\begin{array}{l}\text { No significant change in fasting } \\
\text { ghrelin RYGB group; } \\
\text { Significant decrease in LSG. }\end{array}$ \\
\hline $\begin{array}{l}\text { Oliván et al. } 2009 \\
\text { [39] }\end{array}$ & $\begin{array}{l}\text { Prospective } \\
\text { controlled }\end{array}$ & $\begin{array}{l}21 \text { T2D } \\
10 \text { RYGB } \\
11 \text { diet } \\
9 \text { obese } \\
\text { nondiabetics }\end{array}$ & $\begin{array}{c}47.4 \pm 10.6 \\
42.8 \pm 3.8 \\
45.5 \pm 7.1\end{array}$ & NA & $\begin{array}{l}10 \mathrm{Kg} \text { weight } \\
\text { loss }\end{array}$ & $\begin{array}{l}\text { No change in fasting ghrelin after } \\
\text { RYGB }\end{array}$ \\
\hline $\begin{array}{l}\text { Frühbeck et al. } \\
2004[38]\end{array}$ & $\begin{array}{l}\text { Retrospective } \\
\text { controlled }\end{array}$ & $\begin{array}{l}6 \text { RYGB } \\
7 \text { AGB } \\
3 \text { BPD }\end{array}$ & $\begin{array}{l}42.6 \pm 1.6 \\
45.6 \pm 1.8 \\
60.5 \pm 7.3\end{array}$ & $\begin{array}{l}50.1 \pm 4.4 \\
42.2 \pm 3.1 \\
54.2 \pm 4.3\end{array}$ & $\begin{array}{l}6.1 \pm 0.4 \mathrm{mo} \\
7.0 \pm 0.6 \mathrm{mo} \\
4.4 \pm 0.8 \mathrm{mo}\end{array}$ & $\begin{array}{l}\text { Significant decrease in fasting } \\
\text { ghrelin in RYGB group compared } \\
\text { to the other } 2 \text { groups }\end{array}$ \\
\hline $\begin{array}{l}\text { Foschi et al. } 2008 \\
{[36]}\end{array}$ & $\begin{array}{l}\text { Retrospective } \\
\text { controlled }\end{array}$ & $\begin{array}{l}10 \mathrm{RYGB}(9 \mathrm{~F}) \\
12 \mathrm{VBG}(11 \mathrm{~F})\end{array}$ & $\begin{array}{l}44.1 \pm 1.8 \\
42.9 \pm 1.6\end{array}$ & 20 & $\begin{array}{c}20 \% \\
\text { reduction in } \\
\text { BMI } \\
(=131 \pm 6 \mathrm{~d} \\
\text { for } \mathrm{RYGB}) \\
(119 \pm 4.2 \text { for } \\
\mathrm{VBG})\end{array}$ & $\begin{array}{l}\text { Basal ghrelin plasma levels } \\
\text { reduced after RYGBP but } \\
\text { increased after VBG }\end{array}$ \\
\hline $\begin{array}{l}\text { Rodieux et al. } 2008 \\
{[41]}\end{array}$ & $\begin{array}{l}\text { Cross- } \\
\text { sectional } \\
\text { controlled }\end{array}$ & $\begin{array}{l}8 \text { RYGB } \\
6 \text { GB } \\
8 \text { weight } \\
\text { matched }\end{array}$ & $\begin{array}{l}44.9 \pm 1.8 \\
41.1 \pm 0.5 \\
29.2 \pm 0.8\end{array}$ & $\begin{array}{l}47.8 \pm 3.3 \\
32.4 \pm 2.0\end{array}$ & $\begin{array}{l}9 \text { to } 48 \mathrm{mo} \\
25 \text { to } 85 \mathrm{mo}\end{array}$ & $\begin{array}{l}\text { No change in fasting ghrelin } \\
\text { Maximal PP suppression of } \\
\text { ghrelin greatest in RYGB group }\end{array}$ \\
\hline
\end{tabular}

Abbreviations: ABG: adjustable gastric banding, ARS: anti-reflux surgery, Conv: conventional weight loss, GB: gastric banding, GI: gastrointestinal, IGT: impaired glucose tolerance, NA: data not available, LSG: laparoscopic sleeve gastrectomy, Postop: postoperatively, RYGB: Roux-en-y gastric bypass, T2D: type 2 diabetes, VBG: vertical banded gastroplasty.

a\% EWL: excess weight loss $=[$ (operative weight - follow-up weight $) /$ operative excess weight $] \times 100$. 
TABLE 2: GLP-1 and RYGB surgery.

\begin{tabular}{|c|c|c|c|c|c|c|}
\hline Author/year & Type of study & Subjects & Preop BMI & \% weight loss & $\mathrm{F} / \mathrm{U}$ time & Change in hormone \\
\hline $\begin{array}{l}\text { Morínigo et al. } \\
2006[51]\end{array}$ & $\begin{array}{l}\text { Prospective } \\
\text { controlled }\end{array}$ & $\begin{array}{l}9(7 \mathrm{~F}) \text { RYGB } \\
\text { non diabetic } \\
6 \text { obese }(4 \mathrm{~F})\end{array}$ & $\begin{array}{l}47.4 \pm 6.1 \\
43.6 \pm 7.9\end{array}$ & NA & $6 \mathrm{wk}$ & $\begin{array}{l}\text { Greater increase in active GLP-1 } \\
\text { postmeal in RYGB group postop } \\
\text { compared to weight-matched } \\
\text { obese }\end{array}$ \\
\hline $\begin{array}{l}\text { Laferrère et al. } \\
2007[50]\end{array}$ & $\begin{array}{l}\text { Prospective } \\
\text { controlled }\end{array}$ & $\begin{array}{l}\text { 8F T2DM RYGB } \\
7 \text { non diabetic } \\
\text { obese }\end{array}$ & $\begin{array}{c}43.6 \pm 6.8 \\
37.1 \pm 11.6\end{array}$ & NA & $1 \mathrm{mo}$ & $\begin{array}{l}\text { Fasting- and glucose-stimulated } \\
\text { GLP-1 similar in S and C } \\
1 \text { month after RYGB, increase in } \\
\text { GLP-1 (total and active) in } \\
\text { response to oral glucose }\end{array}$ \\
\hline $\begin{array}{l}\text { Reinehr et al. } 2007 \\
\text { [59] }\end{array}$ & $\begin{array}{l}\text { Prospective } \\
\text { controlled }\end{array}$ & $\begin{array}{c}30 \text { obese }(26 \mathrm{~F}) \\
19 \mathrm{RYGB} \\
11 \mathrm{~GB}\end{array}$ & $45.7 \pm 7.4$ & $50 \%$ & $2 y$ & $\begin{array}{l}\text { Decrease in fasting GLP-1 in } \\
\text { both groups }\end{array}$ \\
\hline $\begin{array}{l}\text { Le Roux et al. } 2007 \\
\text { [56] }\end{array}$ & $\begin{array}{l}\text { Double-blind } \\
\text { randomized } \\
\text { prospective } \\
\text { controlled }\end{array}$ & $\begin{array}{l}7 \text { RYGB } \\
6 \mathrm{AGB}\end{array}$ & $\begin{array}{l}44.5 \pm 2.9 \\
41.9 \pm 7.5\end{array}$ & NA & $\begin{array}{l}9.5 \pm 1.5 \mathrm{mo} \\
17 \pm 1.4 \mathrm{mo}\end{array}$ & $\begin{array}{l}\text { Early }(2 \mathrm{~d}) \text { and increased } \\
\text { responses of PP GLP-1 in RYGB } \\
\text { group only }\end{array}$ \\
\hline $\begin{array}{l}\text { Laferrère et al. } \\
2008 \text { [49] }\end{array}$ & $\begin{array}{l}\text { Prospective } \\
\text { controlled }\end{array}$ & $\begin{array}{l}9 \text { F T2D RYGB } \\
10 \text { F T2D diet } \\
\text { induced weight } \\
\text { loss }\end{array}$ & $\begin{array}{l}43.3 \pm 6.2 \\
43.3 \pm 3.6\end{array}$ & NA & $\begin{array}{l}1 \mathrm{mo} \\
10 \mathrm{Kg} \text { weight } \\
\text { loss }\end{array}$ & $\begin{array}{l}\text { Increase in total GLP-1 after oral } \\
\text { glucose and GLP-1 AUC after } \\
\text { RYGB but not after diet }\end{array}$ \\
\hline $\begin{array}{l}\text { Peterli et al. } 2009 \\
{[54]}\end{array}$ & $\begin{array}{l}\text { Randomized } \\
\text { prospective } \\
\text { controlled }\end{array}$ & $\begin{array}{l}13 \text { RYGB } \\
14 \text { LSG }\end{array}$ & $\begin{array}{l}47 \pm 6.4 \\
45.7 \pm 6.7\end{array}$ & NA & $1 \mathrm{wk}$ and $3 \mathrm{mo}$ & Increased PP GLP-1 RYGB > LSG \\
\hline $\begin{array}{l}\text { Clements et al. } \\
2004[61]\end{array}$ & $\begin{array}{l}\text { Prospective } \\
\text { uncontrolled }\end{array}$ & $\begin{array}{l}20 \text { obese }(15 \mathrm{~F}) \\
\text { with T2D }\end{array}$ & $52.7 \pm 8.8$ & NA & $\begin{array}{l}2,6 \text {, and } 12 \mathrm{wk} \\
\text { postop }\end{array}$ & $\begin{array}{l}\text { No change in fasting GLP- } 1 \text { at } \\
\text { any time point }\end{array}$ \\
\hline $\begin{array}{l}\text { Rubino et al. } 2004 \\
{[60]}\end{array}$ & $\begin{array}{l}\text { Prospective } \\
\text { uncontrolled }\end{array}$ & $\begin{array}{l}\text { S: } 10(9 \mathrm{~F}, 6 \\
\text { T2D) obese } \\
\text { RYGB }\end{array}$ & 46.2 & NA & 3 wk & $\begin{array}{l}\text { No change in fasting GLP-1 in } \\
\text { postop }\end{array}$ \\
\hline $\begin{array}{l}\text { Borg et al. } 2006 \\
\text { [57] }\end{array}$ & $\begin{array}{l}\text { Prospective } \\
\text { uncontrolled }\end{array}$ & 6 RYGB & 48.3 & NA & $\begin{array}{l}1,3,6 \mathrm{mo} \\
\text { postop }\end{array}$ & $\begin{array}{l}\text { PP GLP-1 AUC increased at } 6 \mathrm{mo} \\
\text { postop }\end{array}$ \\
\hline $\begin{array}{l}\text { Morínigo et al. } \\
2006[52]\end{array}$ & $\begin{array}{l}\text { Prospective } \\
\text { uncontrolled }\end{array}$ & $\begin{array}{c}34 \text { RYGB } \\
(23 \mathrm{~F}, 12 \mathrm{NGT} \\
12 \mathrm{IGT}, 10 \mathrm{~T} 2 \mathrm{D})\end{array}$ & $49.1 \pm 1.0$ & $\begin{array}{c}\text { NGT: } \\
34.5 \pm 1.4 \\
\text { IGT: } 29.2 \pm 1.9 \\
\text { DM: } 32.0 \pm 2.4\end{array}$ & $6 \mathrm{wk} 12 \mathrm{mo}$ & $\begin{array}{l}\text { Increase in PP GLP-1 AUC } \\
\text { response in IGT and NGT at } \\
6 \text { wk } \\
\text { Increase in PP GLP-1 AUC } \\
\text { response in all } 3 \text { groups at } 12 \text { mo }\end{array}$ \\
\hline $\begin{array}{l}\text { De Carvalho et al. } \\
2009 \text { [53] }\end{array}$ & $\begin{array}{l}\text { Prospective } \\
\text { uncontrolled }\end{array}$ & $\begin{array}{l}11 \text { NGT }(9 \mathrm{~F}) \\
\text { RYGB } \\
8 \text { AGM }(4 \\
\text { T2DM, } 4 \text { IGT) } \\
\text { (7 F) RYGB }\end{array}$ & $\begin{array}{c}46.1 \pm 2.27 \\
46.5 \pm 2.04 \%\end{array}$ & $\begin{array}{l}39.3 \pm 2.24 \\
36.4 \pm 2.6\end{array}$ & $\begin{array}{c}\text { T1: First } \\
\text { evaluation } \\
\text { T2: presurgery } \\
\text { T3: } 9 \text { mo after } \\
\text { surgery }\end{array}$ & $\begin{array}{l}\text { Increase in GLP-1 levels after } \\
\text { OGTT in both groups at T3 }\end{array}$ \\
\hline $\begin{array}{l}\text { Kashyap et al., } \\
2010[73]\end{array}$ & $\begin{array}{l}\text { Prospective } \\
\text { uncontrolled }\end{array}$ & $\begin{array}{c}16(7 \mathrm{~F}) \mathrm{T} 2 \mathrm{D} \\
9 \mathrm{RYGB} \\
7 \mathrm{GR}\end{array}$ & $47 \pm 9$ & $10 \%$ & $4 \mathrm{wk}$ & $\begin{array}{l}\text { No change in fasting GLP-1 in } \\
\text { both groups } \\
\text { Increase in PP GLP-1 response in } \\
\text { RYGB group only }\end{array}$ \\
\hline $\begin{array}{l}\text { Le Roux et al. } 2006 \\
\text { [55] }\end{array}$ & $\begin{array}{l}\text { Cross- } \\
\text { sectional } \\
\text { controlled }\end{array}$ & $\begin{array}{l}6 \text { RYGB } \\
6 \text { GB } \\
12 \text { obese } \\
15 \text { lean }\end{array}$ & $\begin{array}{l}49.8 \\
46.1 \\
47.1 \\
23.8\end{array}$ & NA & 6 to $36 \mathrm{mo}$ & $\begin{array}{l}\text { Higher postprandial GLP-1 } \\
\text { response in RYGB group } \\
\text { compared to fasting levels and to } \\
\text { other groups }\end{array}$ \\
\hline $\begin{array}{l}\text { Korner et al. } 2007 \\
{[58]}\end{array}$ & $\begin{array}{l}\text { Cross- } \\
\text { sectional } \\
\text { controlled }\end{array}$ & $\begin{array}{l}13 \text { F non } \\
\text { diabetic RYGB } \\
10 \text { F BND } \\
13 \text { F OW }\end{array}$ & $\begin{array}{l}31.3 \pm 1.3 \\
36.1 \pm 1.7 \\
36.1 \pm 2.2\end{array}$ & $\begin{array}{l}35.6 \pm 2.4 \\
24.6 \pm 2.3\end{array}$ & $\begin{array}{l}24.6 \pm 2 \mathrm{mo} \\
\text { postop }\end{array}$ & $\begin{array}{l}\text { Fasting GLP- } 1 \text { similar in all } \\
\text { groups } \\
\text { At } 30 \text { min postmeal, GLP-1 } \\
\text { higher in RYGB group compared } \\
\text { to BND and OW } \\
\text { GLP-1 AUC at } 180 \text { min greater in } \\
\text { RYBG group compared to other } \\
\text { groups }\end{array}$ \\
\hline
\end{tabular}


TABle 2: Continued.

\begin{tabular}{lcccccl}
\hline Author/year & Type of study & Subjects & Preop BMI & \% weight loss & F/U time & Change in hormone \\
\hline \multirow{3}{*}{$\begin{array}{l}\text { Rodieux et al. 2008 } \\
\text { [41] }\end{array}$} & Cross- & 8 RYGB & $44.9 \pm 1.8$ & & 9 to 48 mo & No difference in fasting GLP-1 \\
& sectional & $6 \mathrm{~GB}$ & $41.1 \pm 0.5$ & $47.8 \pm 3.3$ & (RYGB) & between 3 groups; \\
& controlled & 8 weight & $29.2 \pm 0.8$ & $32.4 \pm 2.0$ & 25 to 85 mo & Exaggerated GLP-1 PP \\
& matched & & (GB) & Response in RYGB.
\end{tabular}

Abbreviations: AUC: area under the curve, AGM: abnormal glucose metabolism, BND: adjustable gastric banding, GR: gastric restrictive, IGT: impaired glucose tolerance, LSG: laparoscopic sleeve gastrectomy, NA: data not available, NGT: normal glucose tolerance, OGTT: oral glucose tolerance test, OW: overweight, Postop: postopertaively, PP: postprandial, RYGB: Roux-en-y gastricbBypass, T2D: type 2 diabetes.

TABLE 3: GIP and RYGB surgery.

\begin{tabular}{|c|c|c|c|c|c|c|}
\hline Author/year & Type of study & Subjects & Preop BMI & $\%$ weight loss & $\mathrm{F} / \mathrm{U}$ time & Change in hormone \\
\hline $\begin{array}{l}\text { Laferrère et al. } \\
2007[50]\end{array}$ & $\begin{array}{l}\text { Prospective } \\
\text { controlled }\end{array}$ & $\begin{array}{c}8 \text { F T2D RYGB } \\
7 \text { nondiabetic } \\
\text { obese }\end{array}$ & $\begin{array}{c}43.6 \pm 6.8 \\
37.1 \pm 11.6\end{array}$ & NA & $1 \mathrm{mo}$ & $\begin{array}{l}\text { Fasting- and glucose-stimulated } \\
\text { GIP similar in S and C } \\
1 \text { month after RYGB, increase in } \\
\text { GIP in response to oral glucose. }\end{array}$ \\
\hline $\begin{array}{l}\text { Laferrère et al. } \\
2008 \text { [49] }\end{array}$ & $\begin{array}{l}\text { Prospective } \\
\text { controlled }\end{array}$ & $\begin{array}{l}9 \text { F T2D RYGB } \\
10 \text { F T2D } \\
\text { diet-induced } \\
\text { weight loss }\end{array}$ & $\begin{array}{l}43.3 \pm 6.2 \\
43.3 \pm 3.6\end{array}$ & NA & $\begin{array}{l}1 \mathrm{mo} \\
10 \mathrm{Kg} \text { weight } \\
\text { loss }\end{array}$ & $\begin{array}{l}\text { No change in fasting GIP in both } \\
\text { groups. } \\
\text { Increase in peak GIP after OGTT } \\
\text { in RYGB group only. }\end{array}$ \\
\hline $\begin{array}{l}\text { Rubino et al., } 2004 \\
{[60]}\end{array}$ & $\begin{array}{l}\text { Prospective } \\
\text { uncontrolled }\end{array}$ & $\begin{array}{c}10(9 \mathrm{~F}, 6 \mathrm{~T} 2 \mathrm{D}) \\
\text { obese RYGB }\end{array}$ & 46.2 & NA & $3 \mathrm{wk}$ & $\begin{array}{l}\text { Baseline GIP higher in diabetics } \\
\text { compared to nondiabetics. } \\
\text { Decrease in fasting GIP in } \\
\text { diabetics only. }\end{array}$ \\
\hline $\begin{array}{l}\text { Clements et al. } \\
2004[61]\end{array}$ & $\begin{array}{l}\text { Prospective } \\
\text { uncontrolled }\end{array}$ & $\begin{array}{c}20 \text { obese }(15 \mathrm{~F}) \\
\text { with T2D }\end{array}$ & $52.7 \pm 8.8$ & NA & $\begin{array}{c}2,6 \text { and } \\
12 \text { wk postop }\end{array}$ & $\begin{array}{l}\text { Decrease in fasting GIP at } 6 \text { and } \\
12 \mathrm{wk} \text {. }\end{array}$ \\
\hline $\begin{array}{l}\text { Whitson et al. } 2007 \\
{[72]}\end{array}$ & $\begin{array}{l}\text { Prospective } \\
\text { uncontrolled }\end{array}$ & $\begin{array}{c}10(9 \mathrm{~F}, 5 \mathrm{~T} 2 \mathrm{D}) \\
\text { RYGB }\end{array}$ & $50 \pm 6$ & NA & $6 \mathrm{mo}$ & $\begin{array}{l}\text { No change in GIP postop } \\
\text { (nonfasting). }\end{array}$ \\
\hline $\begin{array}{l}\text { Kashyap et al. } 2010 \\
{[73]}\end{array}$ & $\begin{array}{l}\text { Prospective } \\
\text { uncontrolled }\end{array}$ & $\begin{array}{l}16 \text { (7 females) } \\
\text { T2D } \\
9 \text { RYGB } \\
7 \text { GR }\end{array}$ & $47 \pm 9$ & $10 \%$ & $4 \mathrm{wk}$ & $\begin{array}{l}\text { No change in fasting or PP GIP in } \\
\text { both groups. }\end{array}$ \\
\hline $\begin{array}{l}\text { Korner et al. } 2007 \\
{[58]}\end{array}$ & $\begin{array}{l}\text { Cross- } \\
\text { sectional } \\
\text { controlled }\end{array}$ & $\begin{array}{c}13 \text { F RYGB } \\
10 \text { F BND } \\
13 \text { F overweight }\end{array}$ & $\begin{array}{l}31.3 \pm 1.3 \\
36.1 \pm 1.7 \\
36.1 \pm 2.2\end{array}$ & $\begin{array}{l}35.6 \pm 2.4 \\
24.6 \pm 2.3\end{array}$ & $\begin{array}{l}24.6 \pm 2 \mathrm{mo} \\
\text { postop }\end{array}$ & Blunted PP GIP peak after RYGB \\
\hline
\end{tabular}

Abbreviations: BND: adjustable gastric banding, GR: gastric restrictive, NA: data not available, OGTT: oral glucose tolerance test, Postop: postoperatively, PP: postprandial, RYGB: Roux-en-y gastric bypass, T2D: type 2 diabetes.

OXM on glycemic control in diabetic subjects has not been assessed.

Similar to GLP-1 and PYY, bariatric surgeries that accelerate the delivery of enteral nutrients to distal intestine should result in an increase in OXM levels. One recent study by Laferrère et al. observed a marked increase in OXM levels 1 month after RYGB surgery in morbidly obese women with T2D [79].

4.5. Peptide $Y Y(P Y Y)$. Peptide $Y Y$ is a 36 aminoacid peptide, member of the pancreatic polypeptide family, secreted by the L cells of the terminal ileum and colon postprandially in response to food [80]. It circulates in two endogenous forms: PYY(1-36) and PYY(3-36) with the latter constituting the main circulating form [81]. PYY(3-36) binds to the neuropeptide $\mathrm{Y}$ subtype 2 receptor (NPY2) in the hypothalamus and decreases appetite and food intake as seen in rodents and humans when infused peripherally $[82,83]$. Chronic intravenous administration of PYY in obese rodents resulted in a dose-dependent weight reduction
[84]. PYY(3-36) infusion also decreased ghrelin levels [83]. Furthermore, when a selective NPY2 polyethylene glycolconjugated (PEGylated) peptide agonist was infused in diet-induced obese (DIO) mice, improvements in glucose disposal as well as in plasma insulin and glucose levels were observed [85].

Similar to GLP-1, PYY levels are low in obesity $[86,87]$ and at least a blunted response to a meal has been described in T2D. Levels increase after RYGB surgery in both obese and diabetic subjects and may account for the added beneficial satiety and metabolic effects of this procedure $[35,39,40,51$, $56,57,59,88]$. A summary of the studies is presented in Table S1 (see Table S1 in supplementary materials available online at doi:10.5402/2012/504756).

4.6. Amylin. Amylin is a 37 aminoacid peptide cosecreted with insulin from pancreatic beta cells. It plays a role in glucose homeostasis by slowing gastric emptying [89], suppressing glucagon postprandial secretion and decreasing food intake [90, 91]. Furthermore, amylin has been found to 
have a synergistic effect with leptin on eating, body weight, and body adiposity and a synergistic effect with PYY in controlling food intake $[92,93]$. A state of amylin deficiency has been observed in diabetes as seen in rats with streptozotocininduced B-cell damage and in the spontaneously diabetic BB Wistar rats [94] as well as in humans [95]. Therefore, as with insulin, secretion of amylin requires the presence of functioning beta cells. In parallel to the insulin levels, fasting plasma amylin levels are increased in patients with early type T2D and obesity, suggestive of a state of amylin resistance [96]. Consequently, high amylin levels are expected in obese subjects with T2D, and a reduction in their levels with weight loss should be observed in theory.

Studies supportive have shown that male Sprague Dawley obese rats had a significant reduction in plasma amylin levels after RYGB surgery [97]. Similarly, a decrease in amylin levels was reported by Bose et al. in morbidly obese diabetic women at one month after RYGB surgery [98]. Kashyap et al., however, reported no change in fasting and postprandial amylin levels in obese type 2 diabetic subjects up to 4 weeks after RYGB surgery as shown in Table S2 [73].

4.7. Insulin. Insulin is a 53 aminoacid hormone secreted by the beta cells of the pancreas. It increases uptake of glucose into target cells, promotes formation of glycogen, and decreases gluconeogenesis. A reduction in circulating insulin levels has been observed after RYGB surgery with improved insulin sensitivity as measured by homeostasis model assessment of insulin resistance (HOMA-IR). Improvement in insulin resistance has been reported as early as 6 days after RYGB surgery before any major weight loss has occurred [8]. The mechanism behind the early improvement in insulin resistance remains unclear. Caloric restriction early on after RYGB surgery can decrease hepatic glucose production [99] and reduce skeletal muscle insulin resistance [100]. In addition, the changes in adipocytokine and gut hormones profile that ensue following RYGB surgery act simultaneously to variable extents to improve insulin sensitivity.

\section{Adipokines}

Adipokines are bioactive peptides secreted from adipocytes that have multiple effects on metabolism with currently more than 50 adipokines identified [101]. The effect of these adipocyte-secreted factors on glucose homeostasis has been better elucidated in recent years. Both leptin, one of the first adipokines discovered to influence body fat mass and adiponectin, the most abundant adipocyte-derived protein, have been extensively studied in the regulation of carbohydrate and fat metabolism. Furthermore, favorable changes in their circulating levels after bariatric surgery have been assessed in various studies as described in the following paragraph. Other adipokines such as resistin, visfatin, vaspin, omentin, serum-retinol-binding protein (RBP)-4, chemerin, interleukin (IL)-6, plasminogen activator inhibitor (PAI1), tumor necrosis factor (TNF), alpha, serum amyloid A, and angiotensinogen may have a role in obesity and T2D.
However, data on changes postbariatric surgery are either minimal or nonexistent and were therefore not discussed in this paper.

5.1. Adiponectin. Adiponectin is a 244 aminoacid peptide. It is the most abundant adipokine secreted by the adipose tissue. Reduced levels of adiponectin are seen in obese patients [102]. Adiponectin levels are significantly lower as well in diabetic patients and in those with cardiovascular diseases compared to BMI-matched healthy controls [103, 104]. Studies in several adult populations have shown that adiponectin predicts the development of T2D [105-107]. Adiponectin circulates as three oligomeric isoforms: low molecular weight, medium molecular weight, and high molecular weight isoforms. The HMW adiponectin represents the major active form mediating the favorable metabolic effects of adiponectin [108]. Adiponectin regulates insulin sensitivity by increasing fatty acid oxidation, stimulating glucose uptake, and reducing hepatic gluconeogenesis [109]. An increase in adiponectin levels is observed with weight loss, and this increase is paralleled by an improved insulin resistance [110].

After RYGB surgery, an increase in adiponectin levels has been reported as shown in Table S3 [111-119].

5.2. Leptin. Leptin is a 167 aminoacid peptide secreted primarily by the adipose tissue and circulates at levels proportional to body fat. Leptin regulates appetite, energy expenditure, and body weight [120]. An increase in body fat is associated with an increase in leptin levels that act to decrease food intake. However, the elevated levels of leptin seen in obese individuals do not effectively suppress appetite because of an underlying resistance to the hormone [121]. Theories for leptin resistance suggest a defect in blood brain barrier transport of leptin induced by high-fat diets and abnormalities in leptin receptor signaling [122-124]. The effects of leptin on glucose homeostasis are still unclear. Leptin has been shown to enhance glucose uptake in skeletal muscles, reduce hepatic glucose output, increase fatty acid oxidation, and decrease insulin secretion by pancreatic beta cells [125].

Serum leptin levels have been shown to be reduced after RYGB surgery in several studies as presented in Table S4 $[38,57,60,110,112,115,117,126-130]$. Whether bariatric surgery results in an improvement in the leptin-resistant state remains to be determined. However, the favorable changes in leptin and adiponectin levels after RYGB surgery are similarly seen with weight loss from other bariatric surgery procedures [131] as well as from pharmacological $[132]$ and dietary methods $[133,134]$ suggesting that these changes are more related to fat loss rather than the RYGB surgery itself.

\section{Discussion}

Studies evaluating hormonal changes after RYGB surgery have shown an overall positive change in hormones, favoring 
glycemic control. The orexigenic peptide ghrelin is reduced, while the anorexigenic GLP-1, oxyntomodulin, and PYY are increased. Hormones such as leptin, amylin, GIP, and insulin, to which a suggested state of resistance is observed in obesity and T2D tend to decrease, favor a restored homeostasis. Similarly, a change favoring improved insulin sensitivity with increased adiponectin is seen (Figure 2). A summary of the changes of all the peptides, along with their effect on glycemia and appetite is presented in Figure 3.

Despite the overall findings, there remain certain inconsistencies in the results which can be due to the following factors: some studies lacked an appropriate control group and merely assessed changes before and after RYGB surgery. In the few prospective controlled studies, the follow-up time did not exceed 2 years. As demonstrated in the Swedish Obese Subjects Study (SOS), short-term observations $(<2$ years) cannot mirror the long-term effects of bariatric surgery on comorbidities [135].

Sampling time points varied from one study to another. Some assessed fasting hormonal levels, whereas others measured the hormones in the postprandial state. Furthermore, a standardized meal test is lacking and assays used to measure the various hormones and peptides varied among different studies. For example, as previously mentioned ghrelin exists in two forms: acyl ghrelin that has been shown to produce stimulatory effects on food intake and desacyl ghrelin that induces a state of negative energy balance by inhibiting food intake and delaying gastric emptying [21]. Measuring total, acyl, or desacyl ghrelin will potentially give variable results. Therefore, this confounding factor must be taken into consideration. In addition, other potential explanations include the heterogeneity in the populations studied, variability in the method of reporting weight loss, and variability in the surgical techniques. Specific to changes relating to ghrelin, levels can vary depending on differences in the pouch size as well as configuration particularly if the pouch contains more fundic tissue. Variations in technique are widely noted among different surgeons. They are also noted within the same surgeon's experience as differences in patient's anatomy, body habitus, and effect of prior operations dictate modifications such as lengthening or shortening the pouch $[7,136]$. The stoma diameter is important in determining how fast food is delivered to the small intestine and may play a role in the hormonal changes described above. Moreover, the length of the AL and BPL is not standardized and might have an impact on one or more of the gut incretins. Finally, one important factor not taken into account in most studies is the lack of standardized use of antidiabetic medications which can influence metabolites.

Even after consistent documentation of the hormonal changes, an important question remains in establishing the relationship of these alterations to metabolic control. Is the overall favorable hormonal milieu a result of the negative energy state or a causality of it? It may prove difficult to settle this point. However, controlled studies in the immediate postoperative phase, within one day to one week, would be helpful. Except for one study which measured ghrelin levels as early as two hours postoperatively [34], the vast majority obtain their first measurement two to three

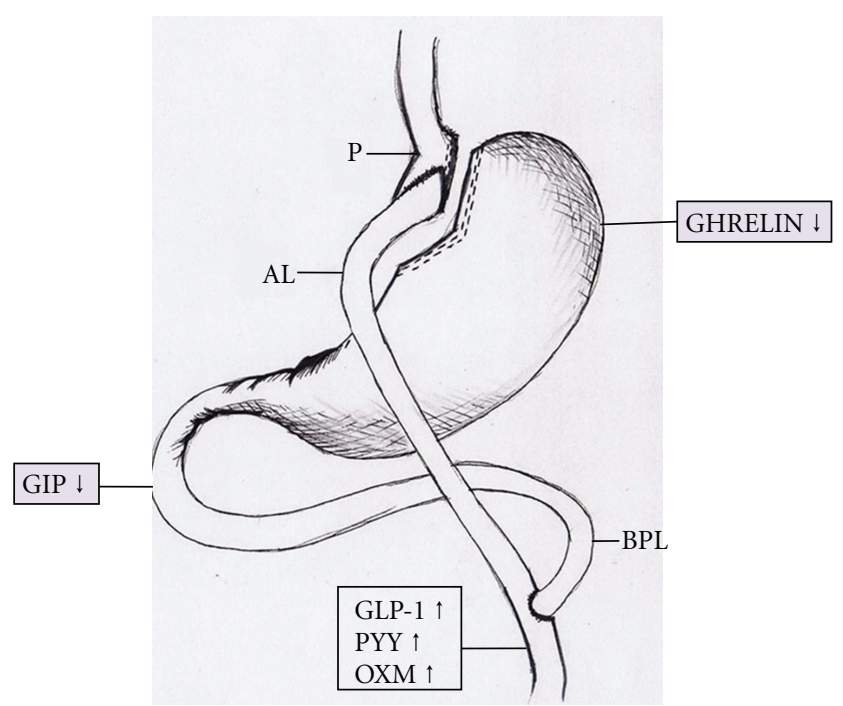

FIGURE 2: Changes in gut-related peptides post-RYGB surgery.

weeks postoperatively. Furthermore, comparison of changes following very low-caloric diets similar in intake to the immediate post-RYGB phase could prove interesting. These are lacking and in practice may be difficult to conduct.

\section{Conclusion}

In summary, in addition to the significant and sustained weight reduction achieved by RYGB surgery, improvement in obesity comorbidities, insulin resistance, and glycemic control is noted. This amelioration is attributed, at least partly, to an alteration in gut peptide release and adipokines. The timing and exact changes of these hormones, as well as their etiologic link to metabolic control postsurgery need to be better established.

Thus, long-term controlled studies and additional research focusing on the very early phase post-RYGB are required for a comprehensive appraisal of the mechanisms behind T2D and its control. These advances will help identify new targets for pharmacological treatment of diabetes.

\section{Abbreviations}

AL: Alimentary limb

BPL: Biliopancreatic limb

DPPIV: Dipeptidyl peptidase IV

GH: $\quad$ Growth hormone

GHSR1: Growth hormone secretagogue receptor 1

GIP: Gastric inhibitory polypeptide

GLP-1: Glucagon-like peptide-1

NPY2: Neuropeptide Y subtype 2

OXM: Oxyntomodulin

PAI-1: Plasminogen activator inhibitor

PYY: $\quad$ Peptide YY

RBP-4: Retinol-binding protein 4

RYGB: Roux-en-y gastric bypass

T2D: $\quad$ Type 2 diabetes. 


\begin{tabular}{|l|l|l|l|l|}
\hline & \multicolumn{1}{|c|}{ Source } & Effect on appetite & Effect on glycemia & Levels post-RYGB \\
\hline Ghrelin & X/A-like cells stomach & increase & increase & decrease \\
\hline GIP & K-cells/duodenum & no effect & increase & conflicting \\
\hline GLP-1 & L-cells/distal ileum & decrease & decrease & increase \\
\hline PYY & L-cells/distal ileum & decrease & decrease & increase \\
\hline OXM & L-cells/distal ileum & decrease & unclear & increase* \\
\hline Amylin & beta cell/pancreas & decrease & decrease & decrease** \\
\hline Insulin & beta cell/pancreas & decrease & decrease & decrease** \\
\hline Leptin & Adipose tissue & decrease & unclear & decrease** \\
\hline Adiponectin & Adipose tissue & no effect & decrease & increase \\
\hline
\end{tabular}

Figure 3: Summary of changes in peptides after RYGB surgery and their effects on glycemia and appetite. ${ }^{*}$ Very few studies. ${ }^{* *}$ Hormonal levels decrease but glycemia improves due to improved sensitivity.

\section{References}

[1] Obesity and Overweight, 2011, http://www.who.int/mediacentre/factsheets/fs311/en/.

[2] R. Steinbrook, "Surgery for severe obesity," The New England Journal of Medicine, vol. 350, no. 11, pp. 1075-1079, 2004.

[3] J. H. Pinkney, C. D. Sjöström, and E. A. M. Gale, "Should surgeons treat diabetes in severely obese people?" The Lancet, vol. 357, no. 9265, pp. 1357-1359, 2001.

[4] H. Buchwald, Y. Avidor, E. Braunwald et al., "Bariatric surgery: a systematic review and meta-analysis," JAMA, vol. 292, no. 14, pp. 1724-1737, 2004.

[5] H. Buchwald, R. Estok, K. Fahrbach et al., "Weight and type 2 diabetes after bariatric surgery: systematic review and metaanalysis," American Journal of Medicine, vol. 122, no. 3, pp. 248-256, 2009.

[6] L. C. Do Rego Furtado, "Procedure and outcomes of Rouxen-Y gastric bypass," British Journal of Nursing, vol. 19, no. 5, pp. 307-313, 2010.

[7] H. Buchwald and J. N. Buchwald, "Evolution of operative procedures for the management of morbid obesity 19502000," Obesity Surgery, vol. 12, no. 5, pp. 705-717, 2002.

[8] K. Wickremesekera, G. Miller, T. DeSilva Naotunne, G. Knowles, and R. S. Stubbs, "Loss of insulin resistance after Roux-en-Y gastric bypass surgery: a time course study," Obesity Surgery, vol. 15, no. 4, pp. 474-481, 2005.

[9] D. E. Kelley, R. Wing, C. Buonocore, J. Sturis, K. Polonsky, and M. Fitzsimmons, "Relative effects of calorie restriction and weight loss in noninsulin- dependent diabetes mellitus," The Journal of Clinical Endocrinology and Metabolism, vol. 77, no. 5, pp. 1287-1293, 1993.

[10] R. R. Henry, L. Scheaffer, and J. M. Olefsky, "Glycemic effects of intensive caloric restriction and isocaloric refeeding in noninsulin-dependent diabetes mellitus," The Journal of Clinical Endocrinology and Metabolism, vol. 61, no. 5, pp. 917-925, 1985.

[11] D. E. Cummings, J. Overduin, and K. E. Foster-Schubert, "Gastric bypass for obesity: mechanisms of weight loss and diabetes resolution," The Journal of Clinical Endocrinology and Metabolism, vol. 89, no. 6, pp. 2608-2615, 2004.

[12] E. Karra, A. Yousseif, and R. L. Batterham, "Mechanisms facilitating weight loss and resolution of type 2 diabetes following bariatric surgery," Trends in Endocrinology and Metabolism, vol. 21, no. 6, pp. 337-344, 2010.

[13] D. E. Cummings, "Endocrine mechanisms mediating remission of diabetes after gastric bypass surgery," International Journal of Obesity, vol. 33, supplement 1, pp. S33-S40, 2009.

[14] F. K. Knop, "Resolution of type 2 diabetes following gastric bypass surgery: involvement of gut-derived glucagon and glucagonotropic signalling?" Diabetologia, vol. 52, no. 11, pp. 2270-2276, 2009.

[15] F. Rubino, A. Forgione, D. E. Cummings et al., "The mechanism of diabetes control after gastrointestinal bypass surgery reveals a role of the proximal small intestine in the pathophysiology of type 2 diabetes," Annals of Surgery, vol. 244, no. 5, pp. 741-749, 2006.

[16] Y. Date, M. Kojima, H. Hosoda et al., "Ghrelin, a novel growth hormone-releasing acylated peptide, is synthesized in a distinct endocrine cell type in the gastrointestinal tracts of rats and humans," Endocrinology, vol. 141, no. 11, pp. 42554261, 2000.

[17] D. E. Cummings, J. Q. Purnell, R. S. Frayo, K. Schmidova, B. E. Wisse, and D. S. Weigle, "A preprandial rise in plasma ghrelin levels suggests a role in meal initiation in humans," Diabetes, vol. 50, no. 8, pp. 1714-1719, 2001.

[18] H. Hosoda, M. Kojima, H. Matsuo, and K. Kangawa, "Ghrelin and des-acyl ghrelin: two major forms of rat ghrelin peptide in gastrointestinal tissue," Biochemical and Biophysical Research Communications, vol. 279, no. 3, pp. 909-913, 2000.

[19] A. M. Wren, C. J. Small, H. L. Ward et al., "The novel hypothalamic peptide ghrelin stimulates food intake and growth hormone secretion," Endocrinology, vol. 141, no. 11, pp. 4325-4328, 2000.

[20] F. Broglio, C. Gottero, F. Prodam et al., "Non-acylated ghrelin counteracts the metabolic but not the neuroendocrine 
response to acylated ghrelin in humans," The Journal of Clinical Endocrinology and Metabolism, vol. 89, no. 6, pp. 3062-3065, 2004.

[21] A. Asakawa, A. Inui, M. Fujimiya et al., "Stomach regulates energy balance via acylated ghrelin and desacyl ghrelin," Gut, vol. 54, no. 1, pp. 18-24, 2005.

[22] K. Takaya, H. Ariyasu, N. Kanamoto et al., "Ghrelin strongly stimulates growth hormone $(\mathrm{GH})$ release in humans," The Journal of Clinical Endocrinology and Metabolism, vol. 85, no. 12, pp. 4908-4911, 2000.

[23] N. Nagaya, M. Kojima, M. Uematsu et al., "Hemodynamic and hormonal effects of human ghrelin in healthy volunteers," American Journal of Physiology, vol. 280, no. 5, pp. R1483-R1487, 2001.

[24] V. Ott, M. Fasshauer, A. Dalski et al., "Direct peripheral effects of ghrelin include suppression of adiponectin expression," Hormone and Metabolic Research, vol. 34, no. 11-12, pp. 640-645, 2002.

[25] M. Murata, Y. Okimura, K. Iida et al., "Ghrelin modulates the downstream molecules of insulin signaling in hepatoma cells," The Journal of Biological Chemistry, vol. 277, no. 7, pp. 5667-5674, 2002.

[26] S. Gnanapavan, B. Kola, S. A. Bustin et al., "The tissue distribution of the mRNA of ghrelin and subtypes of its receptor, GHS-R, in humans," The Journal of Clinical Endocrinology and Metabolism, vol. 87, no. 6, pp. 2988-2991, 2002.

[27] M. Volante, E. Allìa, P. Gugliotta et al., "Expression of ghrelin and of the GH secretagogue receptor by pancreatic islet cells and related endocrine tumors," The Journal of Clinical Endocrinology and Metabolism, vol. 87, no. 3, pp. 1300-1308, 2002.

[28] F. Broglio, E. Arvat, A. Benso et al., "Ghrelin, a natural gh secretagogue produced by the stomach, induces hyperglycemia and reduces insulin secretion in humans," The Journal of Clinical Endocrinology and Metabolism, vol. 86, no. 10, pp. 5083-5086, 2001.

[29] S. S. Damjanovic, N. M. Lalic, P. M. Pesko et al., "Acute effects of ghrelin on insulin secretion and glucose disposal rate in gastrectomized patients," The Journal of Clinical Endocrinology and Metabolism, vol. 91, no. 7, pp. 2574-2581, 2006.

[30] A. C. Heijboer, A. M. van den Hoek, E. T. Parlevliet et al., "Ghrelin differentially affects hepatic and peripheral insulin sensitivity in mice," Diabetologia, vol. 49, no. 4, pp. 732-738, 2006.

[31] R. Barazzoni, M. Zanetti, M. R. Cattin et al., "Ghrelin enhances in vivo skeletal muscle but not liver AKT signaling in rats," Obesity, vol. 15, no. 11, pp. 2614-2623, 2007.

[32] E. Lin, N. Gletsu, K. Fugate et al., "The effects of gastric surgery on systemic ghrelin levels in the morbidly obese," Archives of Surgery, vol. 139, no. 7, pp. 780-784, 2004.

[33] B. Geloneze, M. A. Tambascia, V. F. Pilla, S. R. Geloneze, E. M. Repetto, and J. C. Pareja, "Ghrelin: a gut-brain hormone. Effect of gastric bypass surgery," Obesity Surgery, vol. 13, no. 1, pp. 17-22, 2003.

[34] M. E. Couce, D. Cottam, J. Esplen, P. Schauer, and B. Burguera, "Is ghrelin the culprit for weight loss after gastric bypass surgery? A negative answer," Obesity Surgery, vol. 16, no. 7, pp. 870-878, 2006.

[35] R. Morínigo, J. Vidal, A. M. Lacy, S. Delgado, R. Casamitjana, and R. Gomis, "Circulating peptide YY, weight loss, and glucose homeostasis after gastric bypass surgery in morbidly obese subjects," Annals of Surgery, vol. 247, no. 2, pp. 270275, 2008.
[36] D. Foschi, F. Corsi, F. Colombo et al., "Different effects of vertical banded gastroplasty and Roux-en-Y gastric bypass on meal inhibition of ghrelin secretion in morbidly obese patients," Journal of Investigative Surgery, vol. 21, no. 2, pp. 77-81, 2008.

[37] G. Frühbeck, F. Rotellar, J. L. Hernández-Lizoain et al., "Fasting plasma ghrelin concentrations 6 months after gastric bypass are not determined by weight loss or changes in insulinemia," Obesity Surgery, vol. 14, no. 9, pp. 1208-1215, 2004.

[38] G. Frühbeck, A. Diez-Caballero, M. J. Gil et al., "The decrease in plasma ghrelin concentrations following bariatric surgery depends on the functional integrity of the fundus," Obesity Surgery, vol. 14, no. 5, pp. 606-612, 2004.

[39] B. Oliván, J. Teixeira, M. Bose et al., "Effect of weight loss by diet or gastric bypass surgery on peptide YY3-36 levels," Annals of Surgery, vol. 249, no. 6, pp. 948-953, 2009.

[40] S. N. Karamanakos, K. Vagenas, F. Kalfarentzos, and T. K. Alexandrides, "Weight loss, appetite suppression, and changes in fasting and postprandial ghrelin and peptide-YY levels after Roux-en-Y gastric bypass and sleeve gastrectomy: a prospective, double blind study," Annals of Surgery, vol. 247, no. 3, pp. 401-407, 2008.

[41] F. Rodieux, V. Giusti, D. A. D’Alessio, M. Suter, and L. Tappy, "Effects of gastric bypass and gastric banding on glucose kinetics and gut hormone release," Obesity, vol. 16, no. 2, pp. 298-305, 2008.

[42] M. Gutniak, C. Orskov, J. J. Holst, B. Ahren, and S. Efendic, "Antidiabetogenic effect of glucagon-like peptide-1 (7-36)amide in normal subjects and patients with diabetes mellitus," The New England Journal of Medicine, vol. 326, no. 20, pp. 1316-1322, 1992.

[43] L. Farilla, A. Bulotta, B. Hirshberg et al., "Glucagon-like peptide 1 inhibits cell apoptosis and improves glucose responsiveness of freshly isolated human islets," Endocrinology, vol. 144, no. 12, pp. 5149-5158, 2003.

[44] A. Flint, A. Raben, A. Astrup, and J. J. Holst, "Glucagon-like peptide 1 promotes satiety and suppresses energy intake in humans," The Journal of Clinical Investigation, vol. 101, no. 3, pp. 515-520, 1998.

[45] J. J. Holst, "The physiology of glucagon-like peptide 1," Physiological Reviews, vol. 87, no. 4, pp. 1409-1439, 2007.

[46] M. Zander, S. Madsbad, J. L. Madsen, and J. J. Holst, "Effect of 6-week course of glucagon-like peptide 1 on glycaemic control, insulin sensitivity, and $\beta$-cell function in type 2 diabetes: a parallel-group study," The Lancet, vol. 359, no. 9309, pp. 824-830, 2002.

[47] T. Vilsbøll, T. Krarup, S. Madsbad, and J. Holst, "Defective amplification of the late phase insulin response to glucose by gip in obese type ii diabetic patients," Diabetologia, vol. 45, no. 8, pp. 1111-1119, 2002.

[48] T. Vilsbøll, T. Krarup, J. Sonne et al., "Incretin secretion in relation to meal size and body weight in healthy subjects and people with type 1 and type 2 diabetes mellitus," The Journal of Clinical Endocrinology and Metabolism, vol. 88, no. 6, pp. 2706-2713, 2003.

[49] B. Laferrère, J. Teixeira, J. McGinty et al., "Effect of weight loss by gastric bypass surgery versus hypocaloric diet on glucose and incretin levels in patients with type 2 diabetes," The Journal of Clinical Endocrinology and Metabolism, vol. 93, no. 7, pp. 2479-2485, 2008.

[50] B. Laferrère, S. Heshka, K. Wang et al., "Incretin levels and effect are markedly enhanced 1 month after Roux-en-Y 
gastric bypass surgery in obese patients with type 2 diabetes," Diabetes Care, vol. 30, no. 7, pp. 1709-1716, 2007.

[51] R. Morínigo, V. Moizé, M. Musri et al., "Glucagon-like peptide-1, peptide YY, hunger, and satiety after gastric bypass surgery in morbidly obese subjects," The Journal of Clinical Endocrinology and Metabolism, vol. 91, no. 5, pp. 1735-1740, 2006.

[52] R. Morínigo, A. M. Lacy, R. Casamitjana, S. Delgado, R. Gomis, and J. Vidal, "GLP-1 and changes in glucose tolerance following gastric bypass surgery in morbidly obese subjects," Obesity Surgery, vol. 16, no. 12, pp. 1594-1601, 2006.

[53] C. P. De Carvalho, D. M. Marin, A. L. De Souza et al., "GLP-1 and adiponectin: effect of weight loss after dietary restriction and gastric bypass in morbidly obese patients with normal and abnormal glucose metabolism," Obesity Surgery, vol. 19, no. 3, pp. 313-320, 2009.

[54] R. Peterli, B. Wölnerhanssen, T. Peters et al., "Improvement in glucose metabolism after bariatric surgery: comparison of laparoscopic roux-en-Y gastric bypass and laparoscopic sleeve gastrectomy: a prospective randomized trial," Annals of Surgery, vol. 250, no. 2, pp. 234-241, 2009.

[55] C. W. Le Roux, S. J. B. Aylwin, R. L. Batterham et al., "Gut hormone profiles following bariatric surgery favor an anorectic state, facilitate weight loss, and improve metabolic parameters," Annals of Surgery, vol. 243, no. 1, pp. 108-114, 2006.

[56] C. W. Le Roux, R. Welbourn, M. Werling et al., "Gut hormones as mediators of appetite and weight loss after Rouxen-Y gastric bypass," Annals of Surgery, vol. 246, no. 5, pp. 780-785, 2007.

[57] C. M. Borg, C. W. Le Roux, M. A. Ghatei, S. R. Bloom, A. G. Patel, and S. J. B. Aylwin, "Progressive rise in gut hormone levels after Roux-en-Y gastric bypass suggests gut adaptation and explains altered satiety," British Journal of Surgery, vol. 93, no. 2, pp. 210-215, 2006.

[58] J. Korner, M. Bessler, W. Inabnet, C. Taveras, and J. J. Holst, "Exaggerated glucagon-like peptide- 1 and blunted glucosedependent insulinotropic peptide secretion are associated with Roux-en-Y gastric bypass but not adjustable gastric banding," Surgery for Obesity and Related Diseases, vol. 3, no. 6, pp. 597-601, 2007.

[59] T. Reinehr, C. L. Roth, G. H. Schernthaner, H. P. Kopp, S. Kriwanek, and G. Schernthaner, "Peptide YY and glucagonlike peptide-1 in morbidly obese patients before and after surgically induced weight loss," Obesity Surgery, vol. 17, no. 12, pp. 1571-1577, 2007.

[60] F. Rubino, M. Gagner, P. Gentileschi et al., "The early effect of the Roux-en-Y gastric bypass on hormones involved in body weight regulation and glucose metabolism," Annals of Surgery, vol. 240, no. 2, pp. 236-242, 2004.

[61] R. H. Clements, Q. H. Gonzalez, C. I. Long, G. Wittert, and H. L. Laws, "Hormonal changes after Roux-en Y gastric bypass for morbid obesity and the control of type-II diabetes mellitus," American Surgeon, vol. 70, no. 1, pp. 1-4, 2004.

[62] J. Dupre, S. A. Ross, D. Watson, and J. C. Brown, "Stimulation of insulin secretion by gastric inhibitory polypeptide in man," The Journal of Clinical Endocrinology and Metabolism, vol. 37, no. 5, pp. 826-828, 1973.

[63] A. Trümper, K. Trümper, H. Trusheim, R. Arnold, B. Göke, and D. Hörsch, "Glucose-dependent insulinotropic polypeptide is a growth factor for $\beta$ (INS-1) cells by pleiotropic signaling," Molecular Endocrinology, vol. 15, no. 9, pp. 15591570, 2001.

[64] C. W. Chia, O. D. Carlson, W. Kim et al., "Exogenous glucosedependent insulinotropic polypeptide worsens postprandial hyperglycemia in type 2 diabetes," Diabetes, vol. 58, no. 6, pp. 1342-1349, 2009.

[65] F. C. Lynn, N. Pamir, E. H. C. Ng, C. H. S. McIntosh, T. J. Kieffer, and R. A. Pederson, "Defective glucose-dependent insulinotropic polypeptide receptor expression in diabetic fatty Zucker rats," Diabetes, vol. 50, no. 5, pp. 1004-1011, 2001.

[66] R. H. Eckel, W. Y. Fujimoto, and J. D. Brunzell, "Gastric inhibitory polypeptide enhanced lipoprotein lipase activity in cultured preadipocytes," Diabetes, vol. 28, no. 12, pp. 1141-1142, 1979.

[67] J. Oben, L. Morgan, J. Fletcher, and V. Marks, "Effect of the entero-pancreatic hormones, gastric inhibitory polypeptide and glucagon-like polypeptide-1(7-36) amide, on fatty acid synthesis in explants of rat adipose tissue," Journal of Endocrinology, vol. 130, no. 2, pp. 267-272, 1991.

[68] K. Miyawaki, Y. Yamada, H. Yano et al., "Glucose intolerance caused by a defect in the entero-insular axis: a study in gastric inhibitory polypeptide receptor knockout mice," Proceedings of the National Academy of Sciences of the United States of America, vol. 96, no. 26, pp. 14843-14847, 1999.

[69] M. C. Althage, E. L. Ford, S. Wang, P. Tso, K. S. Polonsky, and B. M. Wice, "Targeted ablation of glucose-dependent insulinotropic polypeptide-producing cells in transgenic mice reduces obesity and insulin resistance induced by a high fat diet," The Journal of Biological Chemistry, vol. 283, no. 26, pp. 18365-18376, 2008.

[70] N. Irwin, P. L. McClean, F. P. M. O’Harte, V. A. Gault, P. Harriott, and P. R. Flatt, "Early administration of the glucosedependent insulinotropic polypeptide receptor antagonist (Pro3)GIP prevents the development of diabetes and related metabolic abnormalities associated with genetically inherited obesity in ob/ob mice," Diabetologia, vol. 50, no. 7, pp. 15321540, 2007.

[71] P. R. Flatt, “Dorothy hodgkin lecture 2008 gastric inhibitory polypeptide (GIP) revisited: a new therapeutic target for obesity-diabetes?" Diabetic Medicine, vol. 25, no. 7, pp. 759764, 2008.

[72] B. A. Whitson, D. B. Leslie, T. A. Kellogg et al., "Enteroendocrine changes after gastric bypass in diabetic and nondiabetic patients: a preliminary study," Journal of Surgical Research, vol. 141, no. 1, pp. 31-39, 2007.

[73] S. R. Kashyap, S. Daud, K. R. Kelly et al., "Acute effects of gastric bypass versus gastric restrictive surgery on $\beta$-cell function and insulinotropic hormones in severely obese patients with type 2 diabetes," International Journal of Obesity, vol. 34, no. 3, pp. 462-471, 2010.

[74] M. A. Ghatei, L. O. Uttenthal, and N. D. Christofides, "Molecular forms of human enteroglucagon in tissue and plasma: plasma responses to nutrient stimuli in health and in disorders of the upper gastrointestinal tract," The Journal of Clinical Endocrinology and Metabolism, vol. 57, no. 3, pp. 488-495, 1983.

[75] C. L. Dakin, C. J. Small, R. L. Batterham et al., "Peripheral oxyntomodulin reduces food intake and body weight gain in rats," Endocrinology, vol. 145, no. 6, pp. 2687-2695, 2004.

[76] B. Schjoldager, P. E. Mortensen, J. Myhre, J. Christiansen, and J. J. Holst, "Oxyntomodulin from distal gut. Role in regulation of gastric and pancreatic functions," Digestive Diseases and Sciences, vol. 34, no. 9, pp. 1411-1419, 1989.

[77] M. A. Cohen, S. M. Ellis, C. W. Le Roux et al., "Oxyntomodulin suppresses appetite and reduces food intake in humans," The Journal of Clinical Endocrinology and Metabolism, vol. 88, no. 10, pp. 4696-4701, 2003. 
[78] K. Wynne, A. J. Park, C. J. Small et al., "Subcutaneous oxyntomodulin reduces body weight in overweight and obese subjects: a double-blind, randomized, controlled trial," Diabetes, vol. 54, no. 8, pp. 2390-2395, 2005.

[79] B. Laferrère, N. Swerdlow, B. Bawa et al., "Rise of oxyntomodulin in response to oral glucose after gastric bypass surgery in patients with type 2 diabetes," The Journal of Clinical Endocrinology and Metabolism, vol. 95, no. 8, pp. 4072-4076, 2010.

[80] T. E. Adrian, G. L. Ferri, and A. J. Bacarese-Hamilton, "Human distribution and release of a putative new gut hormone, peptide YY," Gastroenterology, vol. 89, no. 5, pp. 1070 1077, 1985.

[81] D. Grandt, M. Schimiczek, C. Beglinger et al., "Two molecular forms of Peptide YY (PYY) are abundant in human blood: characterization of a radioimmunoassay recognizing PYY 136 and PYY 3-36," Regulatory Peptides, vol. 51, no. 2, pp. 151$159,1994$.

[82] R. L. Batterham and S. R. Bloom, "The gut hormone peptide YY regulates appetite," Annals of the New York Academy of Sciences, vol. 994, pp. 162-168, 2003.

[83] R. L. Batterham, M. A. Cohen, S. M. Ellis et al., "Inhibition of food intake in obese subjects by peptide YY3-36," The New England Journal of Medicine, vol. 349, no. 10, pp. 941-948, 2003.

[84] R. A. Pittner, C. X. Moore, S. P. Bhavsar et al., "Effects of PYY[3-36] in rodent models of diabetes and obesity," International Journal of Obesity, vol. 28, no. 8, pp. 963-971, 2004.

[85] A. M. van den Hoek, A. C. Heijboer, E. P. M. Corssmit et al., "PYY3-36 reinforces insulin action on glucose disposal in mice fed a high-fat diet," Diabetes, vol. 53, no. 8, pp. 19491952, 2004.

[86] C. W. Le Roux, R. L. Batterham, S. J. B. Aylwin et al., "Attenuated peptide YY release in obese subjects is associated with reduced satiety," Endocrinology, vol. 147, no. 1, pp. 3-8, 2006.

[87] C. L. Roth, P. J. Enriori, K. Harz, J. Woelfle, M. A. Cowley, and T. Reinehr, "Peptide YY is a regulator of energy homeostasis in obese children before and after weight loss," The Journal of Clinical Endocrinology and Metabolism, vol. 90, no. 12, pp. 6386-6391, 2005.

[88] J. P. Valderas, V. Irribarra, C. Boza et al., "Medical and surgical treatments for obesity have opposite effects on peptide YY and appetite: a prospective study controlled for weight loss," The Journal of Clinical Endocrinology and Metabolism, vol. 95, no. 3, pp. 1069-1075, 2010.

[89] A. A. Young, B. R. Gedulin, and T. J. Rink, "Doseresponses for the slowing of gastric emptying in a rodent model by glucagon-like peptide (7-36) NH2, amylin, cholecystokinin, and other possible regulators of nutrient uptake," Metabolism, vol. 45, no. 1, pp. 1-3, 1996.

[90] W. T. Chance, A. Balasubramaniam, F. S. Zhang, S. J. Wimalawansa, and J. E. Fischer, "Anorexia following the intrahypothalamic administration of amylin," Brain Research, vol. 539, no. 2, pp. 352-354, 1991.

[91] J. E. Morley, J. F. Flood, M. Horowitz, P. M. K. Morley, and M. J. Walter, "Modulation of food intake by peripherally administered amylin," American Journal of Physiology, vol. 267, no. 1, pp. R178-R184, 1994.

[92] J. L. Trevaskis, C. Lei, J. E. Koda, C. Weyer, D. G. Parkes, and J. D. Roth, "Interaction of leptin and amylin in the longterm maintenance of weight loss in diet-induced obese rats," Obesity, vol. 18, no. 1, pp. 21-26, 2010.
[93] J. D. Roth, T. Coffey, C. M. Jodka et al., "Combination therapy with amylin and peptide YY[3-36] in obese rodents: anorexigenic synergy and weight loss additivity," Endocrinology, vol. 148, no. 12, pp. 6054-6061, 2007.

[94] D. Bretherton-Watt, M. A. Ghatei, S. Legon, H. Jamal, K. Suda, and S. R. Bloom, "Depletion of islet amyloid polypeptide in the spontaneously diabetic (BB) Wistar rat," Journal of Molecular Endocrinology, vol. 6, no. 1, pp. 3-7, 1991.

[95] J. E. Koda, M. Fineman, T. J. Rink, G. E. Dailey, D. B. Muchmore, and L. G. Linarelli, "Amylin concentrations and glucose control," The Lancet, vol. 339, no. 8802, pp. 11791180, 1992.

[96] T. R. Pieber, J. Roitelman, Y. Lee, K. L. Luskey, and D. T. Stein, "Direct plasma radioimmunoassay for rat amylin(1-37): concentrations with acquired and genetic obesity," American Journal of Physiology, vol. 267, no. 1, pp. E156E164, 1994.

[97] A. C. Shin, H. Zheng, R. L. Townsend, D. L. Sigalet, and H. R. Berthoud, "Meal-induced hormone responses in a rat model of roux-en-Y gastric bypass surgery," Endocrinology, vol. 151, no. 4, pp. 1588-1597, 2010.

[98] M. Bose, J. Teixeira, B. Olivan et al., "Weight loss and incretin responsiveness improve glucose control independently after gastric bypass surgery," Journal of Diabetes, vol. 2, no. 1, pp. 47-55, 2010.

[99] I. M. Jazet, H. Pijl, M. Frölich, J. A. Romijn, and A. E. Meinders, "Two days of a very low calorie diet reduces endogenous glucose production in obese type 2 diabetic patients despite the withdrawal of blood glucose-lowering therapies including insulin," Metabolism, vol. 54, no. 6, pp. 705-712, 2005.

[100] C. Lara-Castro, B. R. Newcomer, J. Rowell et al., "Effects of short-term very low-calorie diet on intramyocellular lipid and insulin sensitivity in nondiabetic and type 2 diabetic subjects," Metabolism, vol. 57, no. 1, pp. 1-8, 2008.

[101] P. Trayhurn and I. S. Wood, "Adipokines: inflammation and the pleiotropic role of white adipose tissue," British Journal of Nutrition, vol. 92, no. 3, pp. 347-355, 2004.

[102] P. W. Peake, A. D. Kriketos, L. V. Campbell, Y. Shen, and J. A. Charlesworth, "The metabolism of isoforms of human adiponectin: studies in human subjects and in experimental animals," European Journal of Endocrinology, vol. 153, no. 3, pp. 409-417, 2005.

[103] K. Hotta, T. Funahashi, Y. Arita et al., "Plasma concentrations of a novel, adipose-specific protein, adiponectin, in type 2 diabetic patients," Arteriosclerosis, Thrombosis, and Vascular Biology, vol. 20, no. 6, pp. 1595-1599, 2000.

[104] N. Ouchi, S. Kihara, Y. Arita et al., "Novel modulator for endothelial adhesion molecules: adipocyte-derived plasma protein adiponectin," Circulation, vol. 100, no. 25, pp. 24732476, 1999.

[105] K. M. Choi, J. Lee, K. W. Lee et al., "Serum adiponectin concentrations predict the developments of type 2 diabetes and the metabolic syndrome in elderly Koreans," Clinical Endocrinology, vol. 61, no. 1, pp. 75-80, 2004.

[106] R. S. Lindsay, T. Funahashi, R. L. Hanson et al., "Adiponectin and development of type 2 diabetes in the Pima Indian population," The Lancet, vol. 360, no. 9326, pp. 57-58, 2002.

[107] M. Daimon, T. Oizumi, T. Saitoh et al., "Decreased serum levels of adiponectin are a risk factor for the progression to type 2 diabetes in the Japanese population: the Funagata study," Diabetes Care, vol. 26, no. 7, pp. 2015-2020, 2003. 
[108] U. B. Pajvani, X. Du, T. P. Combs et al., "Structure-function studies of the adipocyte-secreted hormone Acrp30/ adiponectin: implications for metabolic regulation and bioactivity," The Journal of Biological Chemistry, vol. 278, no. 11, pp. 9073-9085, 2003.

[109] T. Yamauchi, J. Kamon, Y. Minokoshi et al., "Adiponectin stimulates glucose utilization and fatty-acid oxidation by activating AMP-activated protein kinase," Nature Medicine, vol. 8, no. 11, pp. 1288-1295, 2002.

[110] M. Faraj, P. J. Havel, S. Phélis, D. Blank, A. D. Sniderman, and K. Cianflone, "Plasma acylation-stimulating protein, adiponectin, leptin, and ghrelin before and after weight loss induced by gastric bypass surgery in morbidly obese subjects," The Journal of Clinical Endocrinology and Metabolism, vol. 88, no. 4, pp. 1594-1602, 2003.

[111] A. Serra, M. L. Granada, R. Romero et al., "The effect of bariatric surgery on adipocytokines, renal parameters and other cardiovascular risk factors in severe and very severe obesity: 1-year follow-up," Clinical Nutrition, vol. 25, no. 3, pp. 400-408, 2006.

[112] B. A. Whitson, D. B. Leslie, T. A. Kellogg et al., "Adipokine response in diabetics and nondiabetics following the Rouxen-Y gastric bypass: a preliminary study," Journal of Surgical Research, vol. 142, no. 2, pp. 295-300, 2007.

[113] N. G. De La Torre, M. A. Rubio, E. Bordiú et al., "Effects of weight loss after bariatric surgery for morbid obesity on vascular endothelial growth factor-A, adipocytokines, and insulin," The Journal of Clinical Endocrinology and Metabolism, vol. 93, no. 11, pp. 4276-4281, 2008.

[114] M. A. Trakhtenbroit, J. G. Leichman, M. F. Algahim et al., "Body Weight, Insulin Resistance, and Serum Adipokine Levels 2 Years after 2 Types of Bariatric Surgery," American Journal of Medicine, vol. 122, no. 5, pp. 435-442, 2009.

[115] C. Holdstock, B. E. Engström, M. Öhrvall, L. Lind, M. Sundbom, and F. A. Karlsson, "Ghrelin and adipose tissue regulatory peptides: effect of gastric bypass surgery in obese humans," The Journal of Clinical Endocrinology and Metabolism, vol. 88, no. 7, pp. 3177-3183, 2003.

[116] M. M. Swarbrick, I. T. Austrheim-Smith, K. L. Stanhope et al., "Circulating concentrations of high-molecular-weight adiponectin are increased following Roux-en-Y gastric bypass surgery," Diabetologia, vol. 49, no. 11, pp. 2552-2558, 2006.

[117] E. Lin, L. S. Phillips, T. R. Ziegler et al., "Increases in adiponectin predict improved liver, but not peripheral, insulin sensitivity in severely obese women during weight loss," Diabetes, vol. 56, no. 3, pp. 735-742, 2007.

[118] N. Vilarrasa, J. Vendrell, R. Sánchez-Santos et al., "Effect of weight loss induced by gastric bypass on proinflammatory interleukin-18, soluble tumour necrosis factor- $\alpha$ receptors, C-reactive protein and adiponectin in morbidly obese patients," Clinical Endocrinology, vol. 67, no. 5, pp. 679-686, 2007.

[119] C. C. Coughlin, B. N. Finck, J. C. Eagon et al., "Effect of marked weight loss on adiponectin gene expression and plasma concentrations," Obesity, vol. 15, no. 3, pp. 640-645, 2007.

[120] J. Hims-Hagen, "Physiological roles of the leptin endocrine system: differences between mice and humans," Critical Reviews in Clinical Laboratory Sciences, vol. 36, no. 6, pp. 575655, 1999.

[121] R. V. Considine, M. K. Sinha, M. L. Heiman et al., "Serum immunoreactive-leptin concentrations in normal-weight and obese humans," The New England Journal of Medicine, vol. 334, no. 5, pp. 292-295, 1996.

[122] M. Van Heek, D. S. Compton, C. F. France et al., "Dietinduced obese mice develop peripheral, but not central, resistance to leptin," The Journal of Clinical Investigation, vol. 99, no. 3, pp. 385-390, 1997.

[123] K. El-Haschimi, D. D. Pierroz, S. M. Hileman, C. Bjørbæk, and J.S. Flier, "Two defects contribute to hypothalamic leptin resistance in mice with diet-induced obesity," The Journal of Clinical Investigation, vol. 105, no. 12, pp. 1827-1832, 2000.

[124] W. A. Banks, A. B. Coon, S. M. Robinson et al., "Triglycerides induce leptin resistance at the blood-brain barrier," Diabetes, vol. 53, no. 5, pp. 1253-1260, 2004.

[125] B. O. Yildiz and I. C. Haznedaroglu, "Rethinking leptin and insulin action: therapeutic opportunities for diabetes," International Journal of Biochemistry and Cell Biology, vol. 38, no. 5-6, pp. 820-830, 2006.

[126] A. Molina, J. Vendrell, C. Gutiérrez et al., "Insulin resistance, leptin and TNF- $\alpha$ system in morbidly obese women after gastric bypass," Obesity Surgery, vol. 13, no. 4, pp. 615-621, 2003.

[127] E. Bobbioni-Harsch, P. Morel, O. Huber et al., "Energy economy hampers body weight loss after gastric bypass," The Journal of Clinical Endocrinology and Metabolism, vol. 85, no. 12, pp. 4695-4700, 2000.

[128] S. K. Das, S. B. Roberts, M. A. McCrory et al., "Long-term changes in energy expenditure and body composition after massive weight loss induced by gastric bypass surgery 1-4," American Journal of Clinical Nutrition, vol. 78, no. 1, pp. 2230, 2003.

[129] L. Czupryniak, M. Pawlowski, A. Kumor, D. Szymanski, J. Loba, and J. Strzelczyk, "Predicting maximum Roux-en$\mathrm{Y}$ gastric bypass-induced weight reduction-preoperative plasma leptin or body weight?" Obesity Surgery, vol. 17, no. 2, pp. 162-167, 2007.

[130] M. M. Swarbrick, K. L. Stanhope, I. T. Austrheim-Smith et al., "Longitudinal changes in pancreatic and adipocyte hormones following Roux-en-Y gastric bypass surgery," Diabetologia, vol. 51, no. 10, pp. 1901-1911, 2008.

[131] E. V. Kotidis, G. G. Koliakos, V. G. Baltzopoulos, K. N. Ioannidis, J. G. Yovos, and S. T. Papavramidis, "Serum ghrelin, leptin and adiponectin levels before and after weight loss: comparison of three methods of treatment-a prospective study," Obesity Surgery, vol. 16, no. 11, pp. 1425-1432, 2006.

[132] J.-P. Després, A. Golay, and L. Sjöström, "Effects of rimonabant on metabolic risk factors in overweight patients with dyslipidemia," The New England Journal of Medicine, vol. 353, no. 20, pp. 2121-2134, 2005.

[133] C. Rolland, M. Hession, and I. Broom, "Effect of weight loss on adipokine," Diabetes, Metabolic Syndrome and Obesity, vol. 4, pp. 315-323, 2011.

[134] L. K. Forsythe, J. M. W. Wallace, and M. B. E. Livingstone, "Obesity and inflammation: the effects of weight loss," Nutrition Research Reviews, vol. 21, no. 2, pp. 117-133, 2008.

[135] L. Sjöström, A. K. Lindroos, M. Peltonen et al., "Lifestyle, diabetes, and cardiovascular risk factors 10 years after bariatric surgery," The New England Journal of Medicine, vol. 351, no. 26, pp. 2683-2693, 2004.

[136] E. Akkary, S. Sidani, J. Boonsiri et al., "The paradox of the pouch: prompt emptying predicts improved weight loss after laparoscopic Roux-Y gastric bypass," Surgical Endoscopy and Other Interventional Techniques, vol. 23, no. 4, pp. 790-794, 2009. 


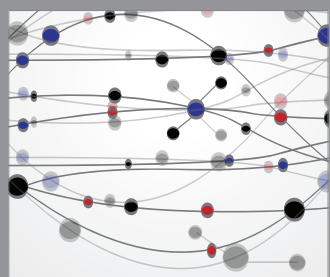

The Scientific World Journal
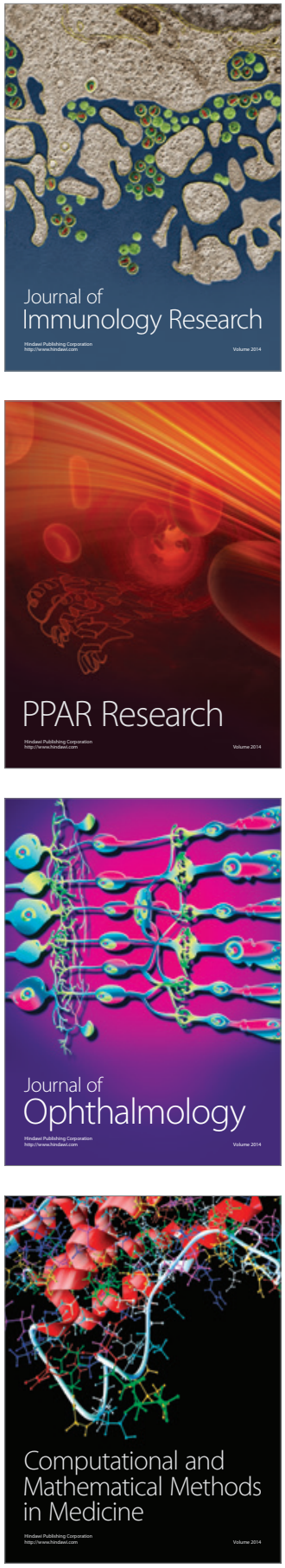

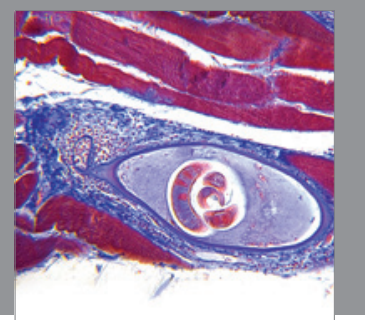

Gastroenterology

Research and Practice
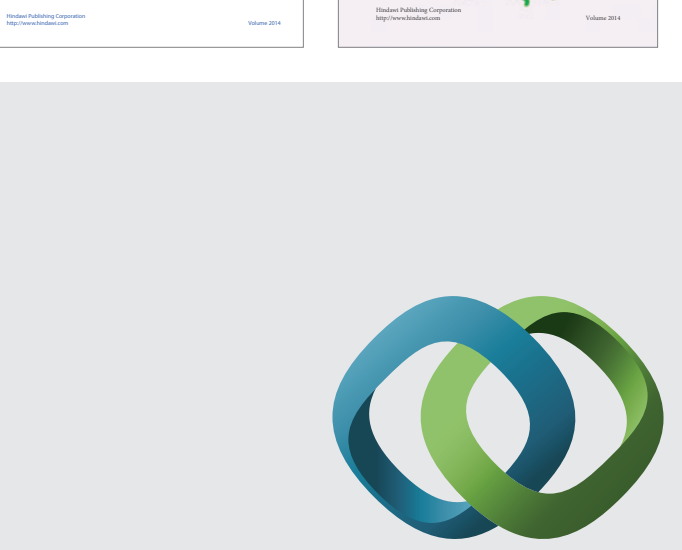

\section{Hindawi}

Submit your manuscripts at

http://www.hindawi.com
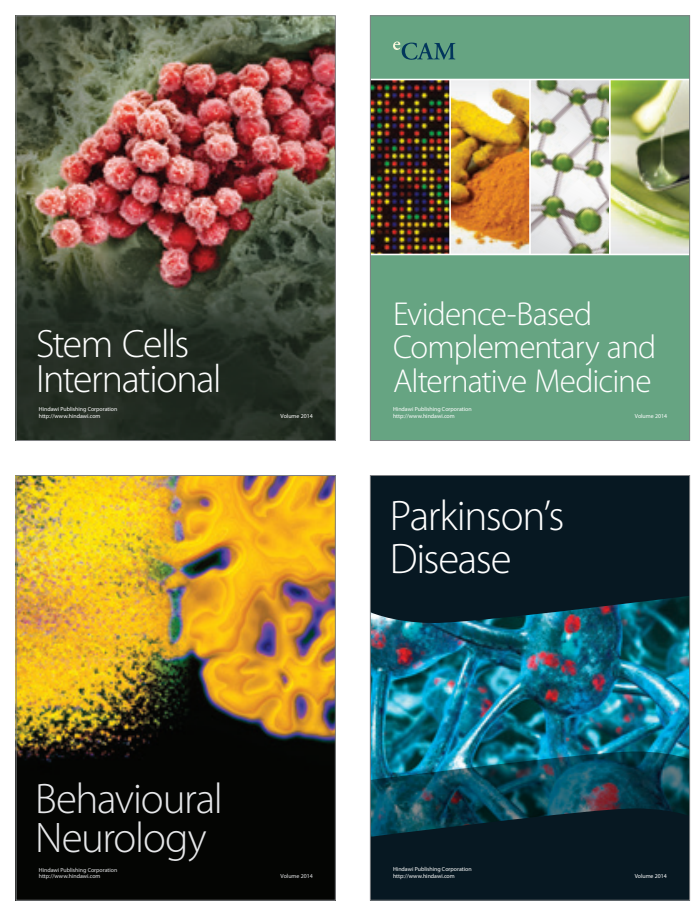

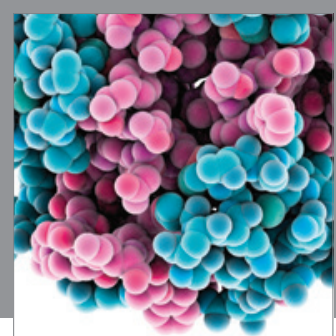

Journal of
Diabetes Research

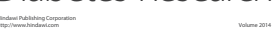

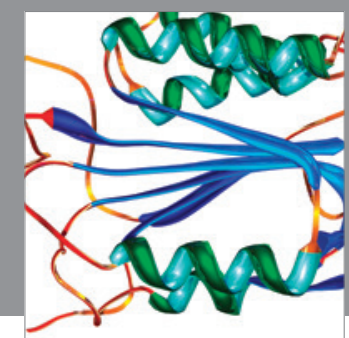

Disease Markers
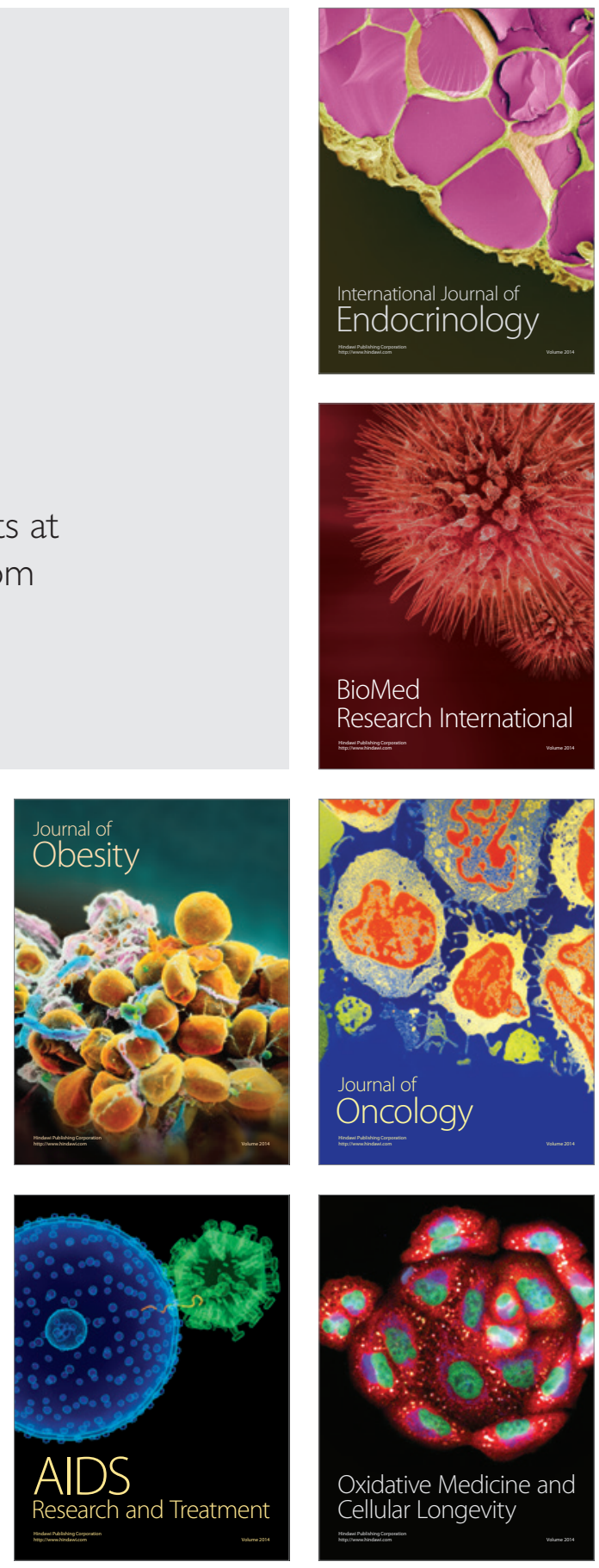